\title{
TYING UP BARIC ALGEBRAS
}

\author{
ANTONIO M. OLLER-MARCÉN*
}

\begin{abstract}
Given two baric algebras $\left(A_{1}, \omega_{1}\right)$ and $\left(A_{2}, \omega_{2}\right)$ we describe a way to define a new baric algebra structure over the vector space $A_{1} \oplus$ $A_{2}$, which we shall denote $\left(A_{1} \bowtie A_{2}, \omega_{1} \bowtie \omega_{2}\right)$. We present some easy properties of this construction and we show that in the commutative and unital case it preserves indecomposability. Algebras of the form $A_{1} \bowtie A_{2}$ in the associative, coutable-dimensional, zero-characteristic case are classified.
\end{abstract}

\section{INTRODUCTION}

Baric algebras play an important role in the theory of genetic algebras. The use of algebraic formalism to study genetic inheritance was introduced by I.M.H. Etherington [3] in the first half of the last century and has revealed fruitful giving rise to many interesting classes of algebras such as train or Bernstein. For a brief survey of this subject we refer to [5] and for an introductory but deeper approach to 6 .

In 1 the notion of decomposable baric algebras was introduced. In the same paper it was also presented a way to construct decomposable baric algebras starting from two baric algebras with an idempotent of weight one. Furthermore in [1] and in 22 the authors analized the indecomposability of some well-known examples of algebras arising in genetics. In this work we define a new way to construct a baric algebra starting from two given baric algebras. Our construction, although similar, is different than that in [1. In particular, while the construction in 1] always gives rise to decomposable baric algebras, we will show that in the commutative unital case our construction preserves indecomposability.

We will also show that baric algebras obtained by our method always have a unique weight homomorphism. Thus, as a consequence, we show that every baric algebra can be embedded in a baric algebra with a unique weight homomorphism.

The paper is organized as follows. The second section presents the construction and the third one gives some properties following easily frome the definition. In the fourth section we study the uniqueness of the weight homomorphism. In the fifth section we study the ideals and focus on the case when our original

2010 Mathematics Subject Classification. Primary 17D92; Secondary 17D99.

Key words and phrases. Baric algebra, Indecomposable baric algebra. 
algebras are commutative and unital, showing that in this case our construction preserves indecomposability. Finally we study the associative case and give a classification when we are in countable dimension and the base field is of characteristic zero.

\section{The construction}

Let $\left(A_{1}, \omega_{1}\right)$ and $\left(A_{2}, \omega_{2}\right)$ be two baric algebras; i.e, $A_{1}$ and $A_{2}$ are algebras over a field $K$ and $\omega_{i}: A_{i} \longrightarrow K$ is a non-zero $K$-algebra homomorphism for $i=1,2$. Now, in the $K$-vector space $A_{1} \oplus A_{2}$ we define a product

$$
\left(a_{1}, a_{2}\right)\left(b_{1}, b_{2}\right)=\left(a_{1} b_{1}+\omega_{2}\left(b_{2}\right) a_{1}, a_{2} b_{2}+\omega_{1}\left(b_{1}\right) a_{2}\right)
$$

which is easily seen to define a $K$-algebra structure on $A_{1} \oplus A_{2}$.

Definition 2.1. Given $\left(A_{i}, \omega_{i}\right)$ with $i=1,2$ two baric algebras we define $A_{1} \bowtie$ $A_{2}$ to be the $K$-vector space $A_{1} \oplus A_{2}$ with the algebra structure given by the product (11).

We can now define an application $\omega_{1} \bowtie \omega_{2}: A_{1} \oplus A_{2} \longrightarrow K$ given by the formula $\omega_{1} \bowtie \omega_{2}\left(a_{1}, a_{2}\right)=\omega_{1}\left(a_{1}\right)+\omega_{2}\left(a_{2}\right)$. Trivially $\omega_{1} \bowtie \omega_{2}$ is $K$-linear and, also, we have that

$$
\begin{aligned}
\omega_{1} \bowtie \omega_{2}\left(\left(a_{1}, a_{2}\right)\left(b_{1}, b_{2}\right)\right) & =\omega_{1} \bowtie \omega_{2}\left(a_{1} b_{1}+\omega_{2}\left(b_{2}\right) a_{1}, a_{2} b_{2}+\omega_{1}\left(b_{1}\right) a_{2}\right) \\
& =\omega_{1}\left(a_{1} b_{1}\right)+\omega_{2}\left(b_{2}\right) \omega_{1}\left(a_{1}\right)+\omega_{2}\left(a_{2} b_{2}\right)+\omega_{1}\left(b_{1}\right) \omega_{2}\left(a_{2}\right) \\
& =\left(\omega_{1}\left(a_{1}\right)+\omega_{2}\left(a_{2}\right)\right)\left(\omega_{1}\left(b_{1}\right)+\omega_{2}\left(b_{2}\right)\right) \\
& =\omega_{1} \bowtie \omega_{2}\left(a_{1}, a_{2}\right) \omega_{1} \bowtie \omega_{2}\left(b_{1}, b_{2}\right) .
\end{aligned}
$$

Thus, $\omega_{1} \bowtie \omega_{2}$ is a $K$-homomorphism and we have the following:

Proposition 2.1. Let $\left(A_{i}, \omega_{i}\right)$ with $i=1,2$ be baric algebras. Then so is the pair $\left(A_{1} \bowtie A_{2}, \omega_{1} \bowtie \omega_{2}\right)$.

Remark. By means of the inclusions $\iota_{i}: A_{i} \hookrightarrow A_{1} \bowtie A_{2}(i=1,2)$ given by $\iota_{i}\left(a_{i}\right)=\left(\delta_{i}^{1} a_{i}, \delta_{i}^{2} a_{i}\right)$ we can see each $A_{i}$ as a subalgebra of $A_{1} \bowtie A_{2}$ and we will identify $A_{i}$ with $\iota_{i}\left(A_{i}\right)$. With this identification it is easy to see that $A_{i} \unlhd_{r} A_{1} \bowtie A_{2}$.

Example 2.1. Let $K$ be a field. Obviously $\left(K, \mathrm{id}_{K}\right)$ is a baric algebra, then $K \bowtie K$ is the vector space $K^{2}$ endowed with the product

$$
(\alpha, \beta)\left(\alpha^{\prime}, \beta^{\prime}\right)=\left(\alpha^{\prime}+\beta^{\prime}\right)(\alpha, \beta) .
$$

In this case we have

$$
\operatorname{id}_{K} \bowtie \operatorname{id}_{K}(\alpha, \beta)=\alpha+\beta .
$$

We will come back to this example later on. 


\section{Some EASY PROPERTIES}

This section is devoted to present some properties arising easily from the previous construction.

We recall that two baric algebras $(A, \omega)$ and $(B, \varphi)$ are said to be isomorphic if there exists a $K$-algebra isomorphism $f: A \longrightarrow B$ such that $\varphi \circ f=\omega$. The following propositions show some nice properties of this construction.

Proposition 3.1. Let $\left(A_{i}, \omega_{i}\right)$ with $i=1,2,3$ be baric algebras. Then we have the following isomorphisms:

(i) $\left(A_{1} \bowtie A_{2}, \omega_{1} \bowtie \omega_{2}\right) \cong\left(A_{2} \bowtie A_{1}, \omega_{2} \bowtie \omega_{1}\right)$.

(ii) $\left(\left(A_{1} \bowtie A_{2}\right) \bowtie A_{3},\left(\omega_{1} \bowtie \omega_{2}\right) \bowtie \omega_{3}\right) \cong\left(A_{1} \bowtie\left(A_{2} \bowtie A_{3}\right), \omega_{1} \bowtie\left(\omega_{2} \bowtie\right.\right.$ $\left.\left.\omega_{3}\right)\right)$.

Proof. Define $f_{1}: A_{1} \bowtie A_{2} \longrightarrow A_{2} \bowtie A_{1}$ by $f\left(a_{1}, a_{2}\right)=\left(a_{2}, a_{1}\right)$, in the same way, define $f_{2}:\left(A_{1} \bowtie A_{2}\right) \bowtie A_{3} \longrightarrow A_{1} \bowtie\left(A_{2} \bowtie A_{3}\right)$ by $f_{2}\left(\left(a_{1}, a_{2}\right), a_{3}\right)=$ $\left(a_{1},\left(a_{2}, a_{3}\right)\right)$. It is easy to see that both maps are weight-preserving $K$-isomorphisms.

Proposition 3.2. Let $\left(A_{1}, \omega_{1}\right),\left(A_{1}^{\prime}, \omega_{1}^{\prime}\right)$ and $\left(A_{2}, \omega_{2}\right)$ be baric algebras and let us suppose that $\left(A_{1}, \omega_{1}\right) \cong\left(A_{1}^{\prime}, \omega_{1}^{\prime}\right)$, then $\left(A_{1} \bowtie A_{2}, \omega_{1} \bowtie \omega_{2}\right) \cong\left(A_{1}^{\prime} \bowtie A_{2}, \omega_{1}^{\prime} \bowtie\right.$ $\left.\omega_{2}\right)$.

Proof. We know by hypothesis that there exists an isomorphism $f: A_{1} \longrightarrow$ $A_{1}^{\prime}$ such that $\omega_{1}^{\prime} \circ f=\omega_{1}$. We can define a map $\tilde{f}: A_{1} \bowtie A_{2} \longrightarrow A_{1}^{\prime} \bowtie$ $A_{2}$ in a natural way by $\widetilde{f}\left(a_{1}, a_{2}\right)=\left(f\left(a_{1}\right), a_{2}\right)$. This map is obviously a $K$ homomorphism and, moreover, $\omega_{1}^{\prime} \bowtie \omega_{2}\left(\tilde{f}\left(a_{1}, a_{2}\right)\right)=\omega_{1}^{\prime}\left(f\left(a_{1}\right)\right)+\omega_{2}\left(a_{2}\right)=$ $\omega_{1}\left(a_{1}\right)+\omega_{2}\left(a_{2}\right)=\omega_{1} \bowtie \omega_{2}\left(a_{1}, a_{2}\right)$ and this completes the proof.

Given a $K$-algebra $A$ and elements $x, y, z \in A$, the definitions of the commutator $[x, y]=x y-y x$ and of the associator $(x, y, z)=(x y) z-x(y z)$ are well known; $A$ being commutative or associative if and only if $[x, y]=0$ for all $x, y \in A$ or $(x, y, z)=0$ for all $x, y, z \in A$ respectively.

Lemma 3.1. Let $\left(A_{i}, \omega_{i}\right)$ for $i=1,2$ be baric algebras. Let $x=\left(a_{1}, a_{2}\right)$ and $y=\left(b_{1}, b_{2}\right)$ be elements of $A_{1} \bowtie A_{2}$. Then we have that:

$$
[x, y]=\left(\left[a_{1}, b_{1}\right]+\omega_{2}\left(b_{2}\right) a_{1}-\omega_{2}\left(a_{2}\right) b_{1},\left[a_{2}, b_{2}\right]+\omega_{1}\left(b_{1}\right) a_{2}-\omega_{1}\left(a_{1}\right) b_{2}\right) .
$$

Recall that the commutative center of an algebra $A$ is the set

$$
\mathcal{K}(A)=\{a \in A \mid[a, b]=0 \forall b \in A\}
$$

In view of the previous lemma, we have the following corollary.

Corollary 3.1. If $\left(A_{i}, \omega_{i}\right)$ with $i=1,2$ are baric algebras, then $\mathcal{K}\left(A_{1} \bowtie A_{2}\right)=0$ 
As usual it is interesting to search for idempotents. In the case of baric algebras we look for idempotents of weight 1 . In this direction we have the following easy result.

Proposition 3.3. Let $\left(A_{i}, \omega_{i}\right)$ for $i=1,2$ be baric $K$-algebras and let $e_{i} \in A_{i}$ be idempotents such that $\omega_{i}\left(e_{i}\right)=1$. Consider the set $\mathfrak{I}=\left\{\left(\lambda e_{1}, \mu e_{2}\right) \mid \lambda+\mu=1\right\}$, then ef $=e$ for all $e, f \in \mathfrak{I}$ and, in particular, $\mathfrak{I}$ consists of idempotents of weight 1.

Proof. $\left(\lambda_{1} e_{1}, \mu_{1} e_{2}\right)\left(\lambda_{2} e_{1}, \mu_{2} e_{2}\right)=\left(\lambda_{1}\left(\lambda_{2}+\mu_{2}\right) e_{1}, \mu_{1}\left(\lambda_{2}+\mu_{2}\right) e_{2}\right)$

\section{UNIQUENESS OF THE WEIGHT HOMOMORPHISM}

In a baric algebra, the weight homomorphism is not uniquely determined in general (see [4] for example). Nevertheless, the following result shows that our construction behaves quite nicely in this sense.

Proposition 4.1. Let $\left(A_{i}, \omega_{i}\right)$ for $i=1,2$ be baric algebras. Then $\omega_{1} \bowtie \omega_{2}$ is uniquely determined.

Proof. Let us suppose that $\varphi: A_{1} \bowtie A_{2} \longrightarrow K$ is a non-trivial homomorphism of $K$-algebras. Then for $i=1,2$ we can define $\varphi_{i}: A_{i} \longrightarrow K$ by $\varphi_{1}\left(a_{1}\right)=\varphi\left(a_{1}, 0\right)$ and $\varphi_{2}\left(a_{2}\right)=\varphi\left(0, a_{2}\right)$. It is easy to check that both $\varphi_{i}$ are $K$-homomorphisms and $\varphi\left(a_{1}, a_{2}\right)=\varphi_{1}\left(a_{1}\right)+\varphi_{2}\left(a_{2}\right)$.

Now, as $\varphi$ is a $K$-homomorphism, $\varphi\left(a_{1}, a_{2}\right) \varphi\left(b_{1}, b_{2}\right)=\varphi\left(\left(a_{1}, a_{2}\right)\left(b_{1}, b_{2}\right)\right)$ and thus, by the preceding considerations:

$$
\left(\varphi_{1}\left(a_{1}\right)+\varphi_{2}\left(a_{2}\right)\right)\left(\varphi_{1}\left(b_{1}\right)+\varphi_{2}\left(b_{2}\right)\right)=\varphi\left(a_{1} b_{1}+\omega_{2}\left(b_{2}\right) a_{1}, a_{2} b_{2}+\omega_{1}\left(b_{1}\right) a_{2}\right)
$$

From this it follows that:

$$
\varphi_{1}\left(a_{1}\right)\left(\omega_{2}\left(b_{2}\right)-\varphi_{2}\left(b_{2}\right)\right)=\varphi_{2}\left(a_{2}\right)\left(\varphi_{1}\left(b_{1}\right)-\omega_{1}\left(b_{1}\right)\right), \forall a_{i}, b_{i} \in A_{i}
$$

So choosing $a_{1} \in \operatorname{Ker} \varphi_{1}$ and $a_{2} \notin \operatorname{Ker} \varphi_{2}$ we have that $\varphi_{1}=\omega_{1}$. Similarly we obtain $\varphi_{2}=\omega_{2}$ and the proof is complete.

As a consequence of this result, together with the fact that $A_{i}$ is a subalgebra of $A_{1} \bowtie A_{2}$ we have the following corollary.

Corollary 4.1. Every baric algebra is a subalgebra of a baric algebra with a unique weight homomorphism.

In [6] it is shown that if a baric algebra $(A, \omega)$ is such that Ker $\omega$ is nil, then the weight homomorphism is uniquely determined. Clearly our construction provides a family of examples showing that the converse is false. 


\section{IDEALS AND INDECOMPOSABILITY.}

Let $\left(A_{i}, \omega_{i}\right)$ with $i=1,2$ be baric algebras, then each $A_{i}$ can be seen as a subalgebra of $A_{1} \bowtie A_{2}$. Now let $I \unlhd_{r} A_{1}$ be a right ideal. We can identify $I$ with $\iota_{1}(I)$ and it is easy to see that with this identification $I \unlhd_{r} A_{1} \bowtie A_{2}$ remains a right ideal.

Now let $I \unlhd_{r} A_{1} \bowtie A_{2}$ be a right ideal. Then we can define

$$
I_{1}=\left\{a_{1} \in A_{1} \mid \exists a_{2} \in A_{2} \text { s.t. }\left(a_{1}, a_{2}\right) \in I\right\}
$$

Again, it is easy to see that $I_{1} \unlhd_{r} A_{1}$ is also a right ideal. Note that if we define the projections $p_{i}: A_{1} \bowtie A_{2} \longrightarrow A_{i}$ in the obvious way, $I_{1}$ is just $p_{1}(I)$. In the same way we can define $I_{2}$.

In view of the previous considerations, it is natural to ask whether an ideal of $A_{i}$ remains an ideal of $A_{1} \bowtie A_{2}$.

Proposition 5.1. Let $I \unlhd A_{i}$ be an ideal. Then $I$ is an ideal of $A_{1} \bowtie A_{2}$ if and only if $I \subseteq K e r \omega_{i}$.

Proof. If $I \unlhd A_{1}$ (the case $I \unlhd A_{2}$ is analogous), then clearly $I \unlhd_{r} A_{1} \bowtie A_{2}$. Now if $x \in I$ and $a_{i} \in A_{i}$ for $i=1,2$ we have that $\left(a_{1}, a_{2}\right)(x, 0)=\left(a_{1} x, \omega_{1}(x) a_{2}\right) \in I$ if and only if $\omega_{1}(x) a_{2}=0$ for all $a_{2} \in A_{2}$. Obviously this happens if and only if $\omega_{1}(x)=0$ and the proof is complete.

While, on the other hand, we have the following:

Proposition 5.2. Let $I \unlhd A_{1} \bowtie A_{2}$ be an ideal such that $I_{1} \neq A_{1}$. Then $I_{1}$ is an ideal of $A_{1}$ if and only if $I_{2} \subseteq K e r \omega_{2}$.

Proof. Given $I \unlhd A_{1} \bowtie A_{2}$ we already know that $I_{1} \unlhd_{r} A_{1}$ is a right ideal. Let us suppose that $I_{2} \subseteq$ Ker $\omega_{2}$, then if $a_{1} \in I_{1}$ and $a \in A_{1}$, there exists $a_{2} \in A_{2}$ such that $\left(a_{1}, a_{2}\right) \in I$; so we have that $(a, 0)\left(a_{1}, a_{2}\right)=\left(a a_{1}, 0\right) \in I$ and this implies that $a a_{1} \in I_{1}$ as desired.

Conversely, suppose that there exists $a_{2} \in I_{2}$ such that $\omega_{2}\left(a_{2}\right) \neq 0$. By definition, there exists $a_{1} \in A_{1}$ such that $\left(a_{1}, a_{2}\right) \in I$; in particular $a_{1} \in I_{1}$ so given any $a \in A_{1}$ we have that $a a_{1} \in I_{1}$. Moreover, $(a, 0)\left(a_{1}, a_{2}\right)=\left(a a_{1}+\right.$ $\left.\omega_{2}\left(a_{2}\right) a, 0\right) \in I$ so $a a_{1}+\omega_{2}\left(a_{2}\right) a \in I_{1}$. Then we have that $\omega_{2}\left(a_{2}\right) a \in I_{1}$ and that $a \in I_{1}$. This implies $A_{1}=I_{1}$, a contradiction.

Remark. If $I \unlhd A_{1} \bowtie A_{2}$ is an ideal, $I \neq A_{1} \bowtie A_{2}$ does not imply $I_{1} \neq A_{1}$. To see this it is enough to consider the ideal $I=\operatorname{Ker} \omega_{1} \bowtie \omega_{2}$, in this case we have that $I_{1}=A_{1}$ although $I \neq A_{1} \bowtie A_{2}$.

Proposition 5.3. Let $\left(A_{i}, \omega_{i}\right)$ for $i=1,2$ be commutative baric algebras and let $I \unlhd A_{1} \bowtie A_{2}$ be an ideal such that $I \subseteq K e r \omega_{1} \bowtie \omega_{2}$. Then $I_{1}=A_{1}$ if and only if $I=K e r \omega_{1} \bowtie \omega_{2}$. 
Proof. Let us suppose $I_{1}=A_{1}$ and choose $a \in A_{1}$ such that $\omega_{1}(a) \neq 0$. Then there exists $b \in A_{2}$ such that $(a, b) \in I$, note that in particular $\omega_{1}(a)=-\omega_{2}(b) \neq$ 0 .

Now take $\left(a_{1}, a_{2}\right) \in \operatorname{Ker} \omega_{1} \bowtie \omega_{2}$, i.e., $\omega_{1}\left(a_{1}\right)+\omega_{2}\left(a_{2}\right)=0$. Being $I \unlhd A_{1} \bowtie A_{2}$ and due to the commutativity of each $A_{i}$ we have:

$$
\begin{aligned}
& (a, b)\left(a_{1}, 0\right)-\left(a_{1}, 0\right)(a, b)=\left(-\omega_{2}(b) a_{1}, \omega_{1}\left(a_{1}\right) b\right) \in I \\
& (a, b)\left(0, a_{2}\right)-\left(0, a_{2}\right)(a, b)=\left(\omega_{2}\left(a_{2}\right) a,-\omega_{1}(a) a_{2}\right) \in I
\end{aligned}
$$

and there are two possible cases:

Firstly, if $\omega_{1}\left(a_{1}\right)=-\omega_{2}\left(a_{2}\right)=0$, then we have

$$
\left(a_{1}, a_{2}\right)=-\left(\omega_{2}(b)\right)^{-1}\left(-\omega_{2}(b) a_{1}, 0\right)-\left(\omega_{1}(a)\right)^{-1}\left(0,-\omega_{1}(a) a_{2}\right) \in I
$$

and secondly, if $\omega_{1}\left(a_{1}\right)=-\omega_{2}\left(a_{2}\right) \neq 0$, then

$$
\left(a_{1}, a_{2}\right)=\left(\omega_{2}(b)\right)^{-1}\left(\left(\omega_{2}\left(a_{2}\right) a,-\omega_{1}(a) a_{2}\right)-\left(-\omega_{2}(b) a_{1}, \omega_{1}\left(a_{1}\right) b\right)+\omega_{1}\left(a_{1}\right)(a, b)\right) \in I
$$

Thus, in both cases $\left(a_{1}, a_{2}\right) \in I$ and the equality holds. The converse was discussed in the previous remark.

Definition 5.1. Let $(A, \omega)$ be a baric algebra. We define the set $\mathcal{I}(A, \omega)$ to be:

$$
\mathcal{I}(A, \omega)=\{I \unlhd A \mid I \subseteq \operatorname{Ker} \omega\}
$$

Proposition 5.4. Let $\left(A_{i}, \omega_{i}\right)$ for $i=1,2$ be commutative unital baric algebras. Then the sets $\mathcal{I}\left(A_{1}, \omega_{1}\right) \times \mathcal{I}\left(A_{2}, \omega_{2}\right)$ and $\mathcal{I}\left(A_{1} \bowtie A_{2}, \omega_{1} \bowtie \omega_{2}\right) \backslash\left\{\operatorname{Ker} \omega_{1} \bowtie \omega_{2}\right\}$ are bijective.

Proof. Let us define maps

$$
\varphi: \mathcal{I}\left(A_{1}, \omega_{1}\right) \times \mathcal{I}\left(A_{2}, \omega_{2}\right) \longrightarrow \mathcal{I}\left(A_{1} \bowtie A_{2}, \omega_{1} \bowtie \omega_{2}\right) \backslash\left\{\operatorname{Ker} \omega_{1} \bowtie \omega_{2}\right\}
$$

and

$$
\psi: \mathcal{I}\left(A_{1} \bowtie A_{2}, \omega_{1} \bowtie \omega_{2}\right) \backslash\left\{\operatorname{Ker} \omega_{1} \bowtie \omega_{2}\right\} \longrightarrow \mathcal{I}\left(A_{1}, \omega_{1}\right) \times \mathcal{I}\left(A_{2}, \omega_{2}\right)
$$

by $\varphi(I, J)=I \bowtie J=\left\{(a, b) \in A_{1} \bowtie A_{2} \mid a \in I, b \in J\right\}$ and $\psi(I)=\left(I_{1}, I_{2}\right)$.

Proposition 5.1 implies that $\varphi$ is well-defined. In the same way Propositions 5.2 and 5.3 imply that $\psi$ is well-defined. Thus, it is enough to see that $\varphi$ and $\psi$ are each other's inverse.

First, let $I_{i} \in \mathcal{I}\left(A_{i}, \omega_{i}\right)$. Then, obviously $\left(I_{1} \bowtie I_{2}\right)_{i}=I_{i}$ and this shows that $\psi \varphi\left(I_{1}, I_{2}\right)=\left(I_{1}, I_{2}\right)$.

On the other hand, let $I \in \mathcal{I}\left(A_{1} \bowtie A_{2}\right) \backslash\left\{\operatorname{Ker} \omega_{1} \bowtie \omega_{2}\right\}$. Clearly $I \subseteq I_{1} \bowtie I_{2}$. Conversely, let $(a, b) \in I_{1} \bowtie I_{2}$. By definition there exists $b^{\prime} \in A_{2}$ such that $\left(a, b^{\prime}\right) \in I$. Since $w_{1}(a)=0$ it follows that $w_{2}\left(b^{\prime}\right)=0$ and, since $I$ is an ideal we have that $(a, 0)=(1,0)\left(a, b^{\prime}\right) \in I$. In the same way $(0, b) \in I$ and we have that $I \subseteq I_{1} \bowtie I_{2}$; i.e., that $\varphi \psi(I)=I$ and the result follows. 
Example 5.1. Let $K$ be any field. We construct $\left(K \bowtie K, \operatorname{id}_{K} \bowtie \operatorname{id}_{K}\right)$ like in Example 2.1. Then a direct application of the previous proposition gives us the simplicity of Ker $\operatorname{id}_{K} \bowtie \operatorname{id}_{K}$.

In [1] the notion of decomposable baric algebra was introduced. Namely, a baric algebra $(A, \omega)$ with an idempotent of weight 1 is decomposable if there are non-trivial ideals $N_{1}$ and $N_{2}$ of $A$, both contained in Ker $\omega$ and such that Ker $\omega=N_{1} \oplus N_{2}$. Otherwise $(A, \omega)$ is indecomposable.

The following result shows that our construction works nicely with respect to indecomposability in the commutative case.

Proposition 5.5. Let $\left(A_{i}, \omega_{i}\right)$ be commutative unital indecomposable baric algebras for $i=1,2$. Then $\left(A_{1} \bowtie A_{2}, \omega_{1} \bowtie \omega_{2}\right)$ is also indecomposable.

Proof. Assume that $\left(A_{1} \bowtie A_{2}, \omega_{1} \bowtie \omega_{2}\right)$ is decomposable. Then there exist ideals $S, T$ such that Ker $\omega_{1} \bowtie \omega_{2}=S \oplus T$. Since both $S, T$ are non-trivial we can apply Proposition 5.4 to get that $S=\left(S_{1}, S_{2}\right)$ and $T=\left(T_{1}, T_{2}\right)$ with $S_{i}, T_{i} \unlhd A_{i}$.

Clearly $S_{i}+T_{i} \subseteq \operatorname{Ker} \omega_{i}$. Now, if $x \in S_{i} \cap T_{i}$ it follows that $(x, 0) \in S \cap T=0$ so $S_{i}$ and $T_{i}$ have direct sum. Moreover, since Ker $\omega_{i} \unlhd A_{1} \bowtie A_{2}$, Ker $\omega_{1} \cap T=$ Ker $\omega_{2} \cap S=0$ it follows that Ker $\omega_{i}=S_{i} \oplus T_{i}$.

If $S_{1}=0$ then it must be $S_{2} \neq 0$. Moreover, $\operatorname{Ker} \omega_{1} \subseteq T_{1}$ and if it was $T_{2}=0$ it follows that Ker $\omega_{2} \subseteq S_{2}$ and Ker $\omega_{1} \oplus \operatorname{Ker} \omega_{2}=\operatorname{Ker} \omega_{1} \bowtie \omega_{2}$ which is false by Proposition 5.4 again. Consequently we have proved that if $S_{1}=0$, then $S_{2}, T_{2} \neq 0$ and $\left(A_{2}, \omega_{2}\right)$ is decomposable.

In the same way it follows that $T_{1}=0$ implies that $\left(A_{2}, \omega_{2}\right)$ is decomposable.

If both $S_{1}$ and $T_{1}$ are non-zero, then $\left(A_{1}, \omega_{1}\right)$ is decomposable and the result follows.

\section{Associativity}

We will start this section with the following lemma:

Lemma 6.1. Let $\left(A_{i}, \omega_{i}\right)$ for $i=1,2$ be baric algebras. Let $x=\left(a_{1}, a_{2}\right)$, $y=\left(b_{1}, b_{2}\right)$ and $z=\left(c_{1}, c_{2}\right)$ be elements of $A_{1} \bowtie A_{2}$. Then we have that:

$(x, y, z)=\left(\left(a_{1}, b_{1}, c_{1}\right)+\omega_{2}\left(b_{2}\right)\left(a_{1} c_{1}-\omega_{1}\left(c_{1}\right) a_{1}\right),\left(a_{2}, b_{2}, c_{2}\right)+\omega_{1}\left(b_{1}\right)\left(a_{2} c_{2}-\omega_{2}\left(c_{2}\right) a_{2}\right)\right)$.

We can use this to prove the following characterization:

Proposition 6.1. Let $\left(A_{i}, \omega_{i}\right)$ with $i=1,2$ be baric algebras. Then the algebra $A_{1} \bowtie A_{2}$ is associative if and only if $\left(a_{1}, a_{2}\right)\left(b_{1}, b_{2}\right)=\left(\omega_{1} \bowtie \omega_{2}\left(b_{1}, b_{2}\right)\right)\left(a_{1}, a_{2}\right)$ for all $\left(a_{1}, a_{2}\right),\left(b_{1}, b_{2}\right) \in A_{1} \bowtie A_{2}$.

Proof. Put $x=\left(a_{1}, a_{2}\right), y=\left(b_{1}, b_{2}\right)$ and $z=\left(c_{1}, c_{2}\right)$. Let us suppose that $A_{1} \bowtie A_{2}$ is associative. Then each $A_{i}$ is also associative because they are 
subalgebras of $A_{1} \bowtie A_{2}$. So, by Lemma 6.1 :

$$
0=(x, y, z)=\left(\omega_{2}\left(b_{2}\right)\left(a_{1} c_{1}-\omega_{1}\left(c_{1}\right) a_{1}\right), \omega_{1}\left(b_{1}\right)\left(a_{2} c_{2}-\omega_{2}\left(c_{2}\right) a_{2}\right)\right) .
$$

and choosing $b_{i} \notin \operatorname{Ker} \omega_{i}$ we have that $a_{i} c_{i}=\omega_{i}\left(c_{i}\right) a_{i}$ for all $a_{i}, c_{i} \in A_{i}$. Thus we have that $\left(a_{1}, a_{2}\right)\left(b_{1}, b_{2}\right)\left(=\omega_{1} \bowtie \omega_{2}\left(b_{1}, b_{2}\right)\right)\left(a_{1}, a_{2}\right)$ and the proof is complete as the converse is just an easy computation.

A $K$-algebra $A$ is called left (resp. right) alternative if $(x, x, y)=0$ for all $x, y \in A$ (resp. $(x, y, y)=0$ for all $x, y \in A)$. We say that $A$ is alternative if it is both left and right alternative. Of course an associative algebra is left and right alternative. As an easy consequence of Lemma 6.1 we have:

Proposition 6.2. Let $\left(A_{i}, \omega_{i}\right)$ with $i=1,2$ be associative baric algebras. Then the following are equivalent:

(i) $A_{1} \bowtie A_{2}$ is associative.

(ii) $A_{1} \bowtie A_{2}$ is left alternative.

(iii) $A_{1} \bowtie A_{2}$ is right alternative.

Example 6.1. Let $K$ be any field. Thanks to Proposition 3.1(ii) and recalling Example 2.1, we can unambiguously define the baric algebra $\left(K^{\bowtie n}, \operatorname{id}_{K}^{\bowtie n}\right)$, where $K^{\bowtie n}$ stands for $K \bowtie \ldots \bowtie K$ and $\operatorname{id}_{K}^{\bowtie n}=\operatorname{id}_{K} \bowtie \ldots \bowtie \operatorname{id}_{K}$ is defined by the formula $\operatorname{id}_{K}^{\bowtie n}\left(\alpha_{1}, \ldots, \alpha_{n}\right)=\alpha_{1}+\cdots+\alpha_{n}$. Then, due to Proposition 6.1, $K^{\bowtie n}$ is associative.

The remaining of this section will be devoted to show that, under certain assumptions, the previous example is the only situation in which our construction is associative.

Let $(A, \omega)$ be a baric algebra over a field $K$ and let us choose $\left\{e_{i} \mid i \in I\right\}$ any $K$-basis for $A$. Put $\epsilon_{i}=\omega\left(e_{i}\right)$ for all $i \in I$ and observe that we can suppose, without loss of generality, that $\epsilon_{i} \in\{0,1\}$ for all $i \in I$. Moreover we have:

Lemma 6.2. Let $K$ be a field with char $K=0$ and let $(A, \omega)$ be a baric $K$ algebra of countable dimension. Then $A$ admits a basis such that every element in the basis is of weight 1.

Proof. Let $\left\{e_{i} \mid i \in I\right\}$ with $|I| \leq \aleph_{0}$ be a $K$-basis of $A$. We can suppose that $I \subseteq \mathbb{N}$ and that $\epsilon_{1}=1$. Now for each $n \in I$ we define $e_{n}^{\prime}=\frac{1}{\sum_{j \leq n} \epsilon_{j}} \sum_{j \leq n} e_{j}$.

Then $\left\{e_{i}^{\prime} \mid i \in I\right\}$ is the desired basis.

Proposition 6.3. Let $K$ be a field with char $K=0$ and let $(A, \omega)$ be a countabledimensional baric $K$-algebra such that $x y=\omega(y) x$ for all $x, y \in A$. Then, if $\nu=\operatorname{dim}_{K} A$, we have $(A, \omega) \cong\left(K^{\bowtie \nu}, i d_{K}^{\bowtie \nu}\right)$ as baric algebras. 
Proof. We consider the $K$-basis of $A\left\{e_{i} \mid i \in I\right\}$ with $\nu=|I| \leq \aleph_{0}$ and $\omega\left(e_{i}\right)=1$ for all $i \in I$ given by the previous lemma. We define $\left(A_{i}, \omega_{i}\right)=\left(K e_{i},\left.\omega\right|_{K e_{i}}\right)$. Obviously $(A, \omega)=\left(\bowtie_{i=1}^{\nu} A_{i}, \bowtie_{i=1}^{\nu} \omega_{i}\right)$ and the proof is complete as $\left(K e_{i},\left.\omega\right|_{K e_{i}}\right) \cong$ $\left(K, \operatorname{id}_{K}\right)$ trivially.

Finally, as a consequence of this proposition we obtain the following:

Corollary 6.1. Let $\left(A_{i}, \omega_{i}\right)$ for $i=1,2$ be countable-dimensional baric $K$ algebras with char $K=0$. Then $A_{1} \bowtie A_{2}$ is associative if and only if $\left(A_{i}, \omega_{i}\right) \cong$ $\left(K^{\bowtie \nu_{i}}, i d_{K}^{\bowtie \nu_{i}}\right)$ with $\nu_{i}=\operatorname{dim}_{K} A_{i}$. In particular, $\left(A_{1} \bowtie A_{2}, \omega_{1} \bowtie \omega_{2}\right) \cong$ $\left(K^{\bowtie \nu}, i d_{K}^{\bowtie \nu}\right)$ with $\nu=\nu_{1}+\nu_{2}$.

\section{REFERENCES}

[1] COSTA R.-GUZZO, H.: Indecomposable baric algebras, Linear Algebra Appl. 183, (1993), 223-236.

[2] COSTA R.-GUZZO, H.: Indecomposable baric algebras II, Linear Algebra Appl. 196, (1994), 233-242.

[3] ETHERINGTON, I. M. H.: Genetic algebras, Proc. Roy. Soc. Edinburgh 59, (1939), $242-258$.

[4] LÓPEZ-SÁNCHEZ, J.-RODRÍGUEZ SANTA MARÍA, E.: Multibaric algebras, In: Non-associative algebra and its applications (S. González, ed.), Math. Appl. 303, Kluwer Acad. Publ.,Dordrecht, 1994, pp. 235-240.

[5] REED, M. L.: Algebraic Structure of genetic inheritance, Bull. Amer. Math. Soc. (N.S.) 34, (1997), 107-130.

[6] WORZ-BUSEKROS, A.: Algebras in genetics, Lecture Notes in Biomath., No. 36, Springer-Verlag, New York, 1980.

* Departamento de Matemáticas

UNIVERSIDAD DE ZARAGOZA

C/ Pedro Cerbuna 12, 50009

ZARAGOZA

SPAIN

E-mail address: oller@unizar.es 